\title{
8 Anhang
}

\subsection{Zweifelsfälle}

Die beiden folgenden Tabellen geben eine Übersicht über Paare von Komposita und Phrasen, die zwar formal äquivalent sind, deren Bedeutungsidentität bzw. Benennungsstatus aber im Material nicht genug war. Sie wurden daher in Kapitel 5.5 nicht berücksichtigt.

Tab. 1: Zweifelsfälle im Deutschen (Primärmaterial)

\begin{tabular}{lll}
\hline Phrase & Kompositum & Kommentar \\
\hline bitter & & \\
bitteres Bier & Bitterbier & Bedeutungsidentität unklar \\
bitterer Trank & Bittertrank & Benennungsstatus der Phrase unsicher \\
bittere Wurzel & Bitterwurzel & Benennungsstatus der Phrase unsicher, \\
& & Bedeutungsidentität unklar \\
\hline
\end{tabular}

\section{fremd}

fremdes Gut

Fremdgut

fremde Sprache

Fremdsprache

fremde Währung

Fremdwährung

fremdes Wort

Fremdwort

Benennungsstatus der Phrase unsicher

Benennungsstatus der Phrase unsicher,

Bedeutungsidentität unklar

Benennungsstatus der Phrase unsicher

Benennungsstatus der Phrase unsicher

(allerdings im DWB!)

\section{geheim}

geheimes Abkommen

geheimes Archiv

geheimer Befehl

geheimes Dokument

geheimer Gang

geheime Gesellschaft

geheime Instruktion

geheime Lehre

geheimes Mittel

geheime Sache

geheimes Schreiben

geheimer Schreiber

geheime Schrift

geheimes Siegel

geheimer Sinn

geheime Sitzung
Geheimabkommen

Geheimarchiv

Geheimbefehl

Geheimdokument

Geheimgang

Geheimgesellschaft

Geheiminstruktion

Geheimlehre

Geheimmittel

Geheimsache

Geheimschreiben

Geheimschreiber

Benennungsstatus der Phrase unsicher

Bedeutungsidentität unklar

Benennungsstatus der Phrase unsicher

Benennungsstatus der Phrase unsicher

Benennungsstatus der Phrase unsicher

Benennungsstatus der Phrase unsicher

Benennungsstatus der Phrase unsicher

Benennungsstatus der Phrase unsicher

Benennungsstatus der Phrase unsicher

Benennungsstatus der Phrase unsicher

Benennungsstatus der Phrase unsicher

Phrase nur als Verweis auf Henisch

(17. Jh.) genannt, daher im Primär-

material keine Konkurrenzvariante

Bedeutungsidentität unklar

Benennungsstatus der Phrase unsicher

Benennungsstatus der Phrase unsicher

Benennungsstatus der Phrase unsicher 


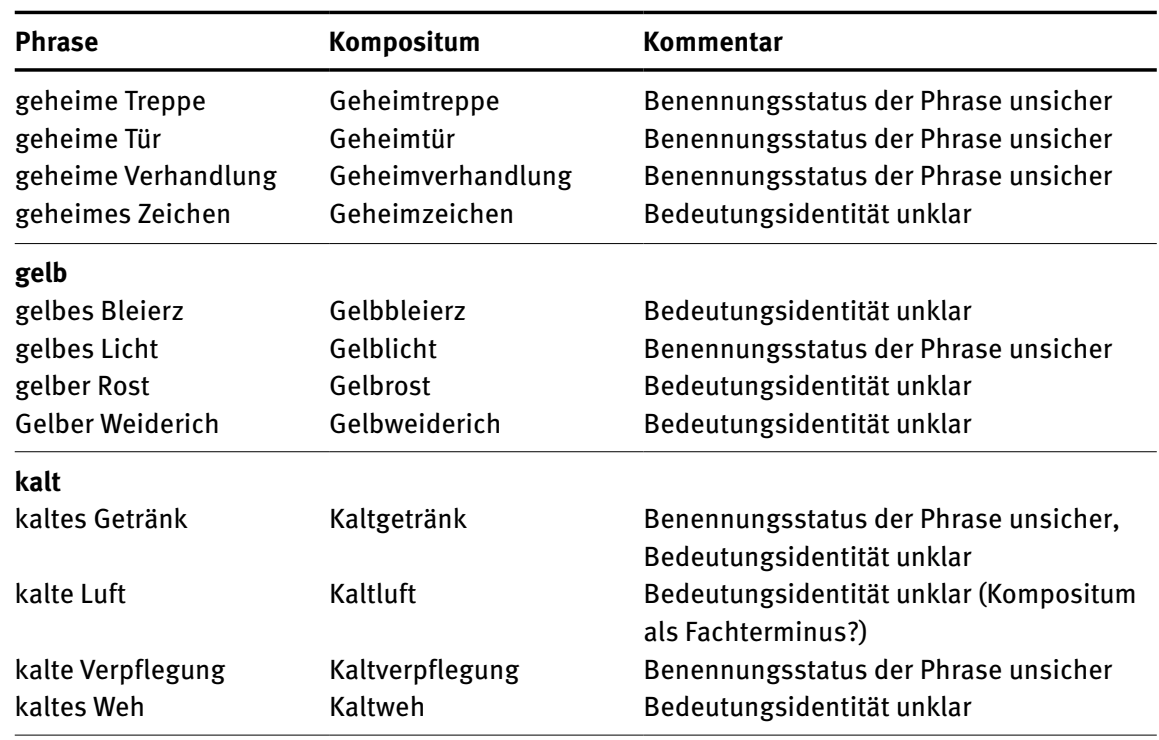

\section{negativ}

negativer Begriff

negative Bilanz

negativer Film

negativer Held

negative Leistung

negative Reaktion

rot

$\begin{array}{ll}\begin{array}{ll}\text { rotes Bleierz } \\ \text { roter Eisenstein } \\ \text { rotes Gebirge } \\ \text { rotes Kupfer }\end{array} & \begin{array}{l}\text { Rotbleierz } \\ \text { Roteisenstein } \\ \text { Rotgebirge } \\ \text { Rotkupfer }\end{array} \\ \text { rote Weide } & \text { Rotweide } \\ \text { roter Wenzel } & \text { Rotwenzel }\end{array}$

\section{schwarz}

$\begin{array}{ll}\text { schwarze Amsel } & \text { Schwarzamsel } \\ \text { schwarzes Geschäft } & \text { Schwarzgeschäft } \\ \text { schwarzer Hafer } & \text { Schwarzhafer } \\ \text { schwarzes Kupfererz } & \text { Schwarzkupfererz } \\ \text { schwarzer Pfeffer } & \text { Schwarzpfeffer } \\ \text { schwarzer Rock } & \text { Schwarzrock } \\ \text { schwarzer Specht } & \text { Schwarzspecht } \\ \text { schwarzer Storch } & \text { Schwarzstorch }\end{array}$

Negativbegriff

Negativbilanz

Negativfilm

Negativheld

Negativleistung

Negativreaktion
Benennungsstatus der Phrase unsicher, Bedeutungsidentität unklar Bedeutungsidentität unklar unterschiedliche Struktur möglich (K: N+N?) Benennungsstatus der Phrase unsicher Benennungsstatus der Phrase unsicher Benennungsstatus der Phrase unsicher
Bedeutungsidentität unklar

Bedeutungsidentität unklar Bedeutungsidentität unklar Benennungsstatus der Phrase unsicher, Bedeutungsidentität unklar Bedeutungsidentität unklar Bedeutungsidentität unklar
Bedeutungsidentität unklar

Benennungsstatus der Phrase unsicher Bedeutungsidentität unklar Bedeutungsidentität unklar Bedeutungsidentität unklar Benennungsstatus der Phrase unsicher Benennungsstatus der Phrase unsicher, Bedeutungsidentität unklar

Benennungsstatus der Phrase unsicher, Bedeutungsidentität unklar 


\begin{tabular}{lll}
\hline Phrase & Kompositum & Kommentar \\
\hline $\begin{array}{l}\text { sozial } \\
(-)\end{array}$ & $(-)$ & $(-)$ \\
\hline still & $(-)$ & $(-)$ \\
\hline $\begin{array}{l}\text { tief } \\
\text { tiefer Schnee }\end{array}$ & $\begin{array}{l}\text { Tiefschnee } \\
\text { tiefe Stimme }\end{array}$ & $\begin{array}{l}\text { Benennungsstatus der Phrase unsicher } \\
\text { Tlefstimme }\end{array}$ \\
\hline $\begin{array}{l}\text { zivil } \\
\text { ziviler Prozess }\end{array}$ & Zivilprozess & Benennungstatus der Phrase unsicher \\
\hline
\end{tabular}

Tab. 2: Zweifelsfälle im Niederländischen (Primärmaterial)

\begin{tabular}{lll}
\hline Phrase & Kompositum & Ko \\
\hline bitter & $(-)$ & $(-)$ \\
$(-)$ & $(-)$ & \\
\hline
\end{tabular}

\section{civiel}
(-)
$(-)$
$(-)$

\section{diep}

$(-) \quad(-)$

geel

gele hars

geelhars

Bedeutungsidentität unklar

gele ijzersteen

geelijzersteen

Bedeutungsidentität unklar

\section{geheim}

(-)

$(-)$

$(-)$

koud

koud zweet

koudzweet

Benennungsstatus unsicher

\section{negatief}

(-)

$(-)$

$(-)$

\section{rood}

rood korral

roodkoraal

Benennungsstatus unsicher

rood krijt

roodkrijt

Bedeutungsidentität unklar

rode lak

roodlak

Benennungsstatus unsicher

\section{sociaal}

(-)

(-)

(-) 


\begin{tabular}{lll}
\hline Phrase & Kompositum & Kommentar \\
\hline $\begin{array}{ll}\text { stil } \\
(-)\end{array}$ & $(-)$ & $(-)$ \\
\hline $\begin{array}{l}\text { vreemd } \\
(-)\end{array}$ & $(-)$ & $(-)$ \\
\hline $\begin{array}{l}\text { zwart } \\
\text { zwarte baars } \\
\text { zwarte vos }\end{array}$ & $\begin{array}{l}\text { zwartbaars } \\
\text { zwartvos }\end{array}$ & $\begin{array}{l}\text { Bedeutungsidentität unklar } \\
\text { Bedeutungsidentität unklar }\end{array}$ \\
\hline
\end{tabular}

\subsection{Chronologische Übersicht konkurrierender Dubletten}

Die folgenden Tabellen dienen als Grundlage für die in Kapitel 5.5 beschriebenen Konkurrenzen von Dublettenpaaren (hier allerdings ausschließlich für Bildungen mit genuin qualitativen Adjektiven). Sie geben jeweils für das Deutsche bzw. Niederländische an, in welchen Wörterbüchern des Primärmaterials Dubletten verzeichnet sind, und erlauben durch die visuelle Darstellung eine schnelle Übersicht über die Entwicklung konkurrierender Phrasen und Komposita. 
Chronologische Übersicht zu konkurrierenden Dubletten im Primärmaterial des Deutschen

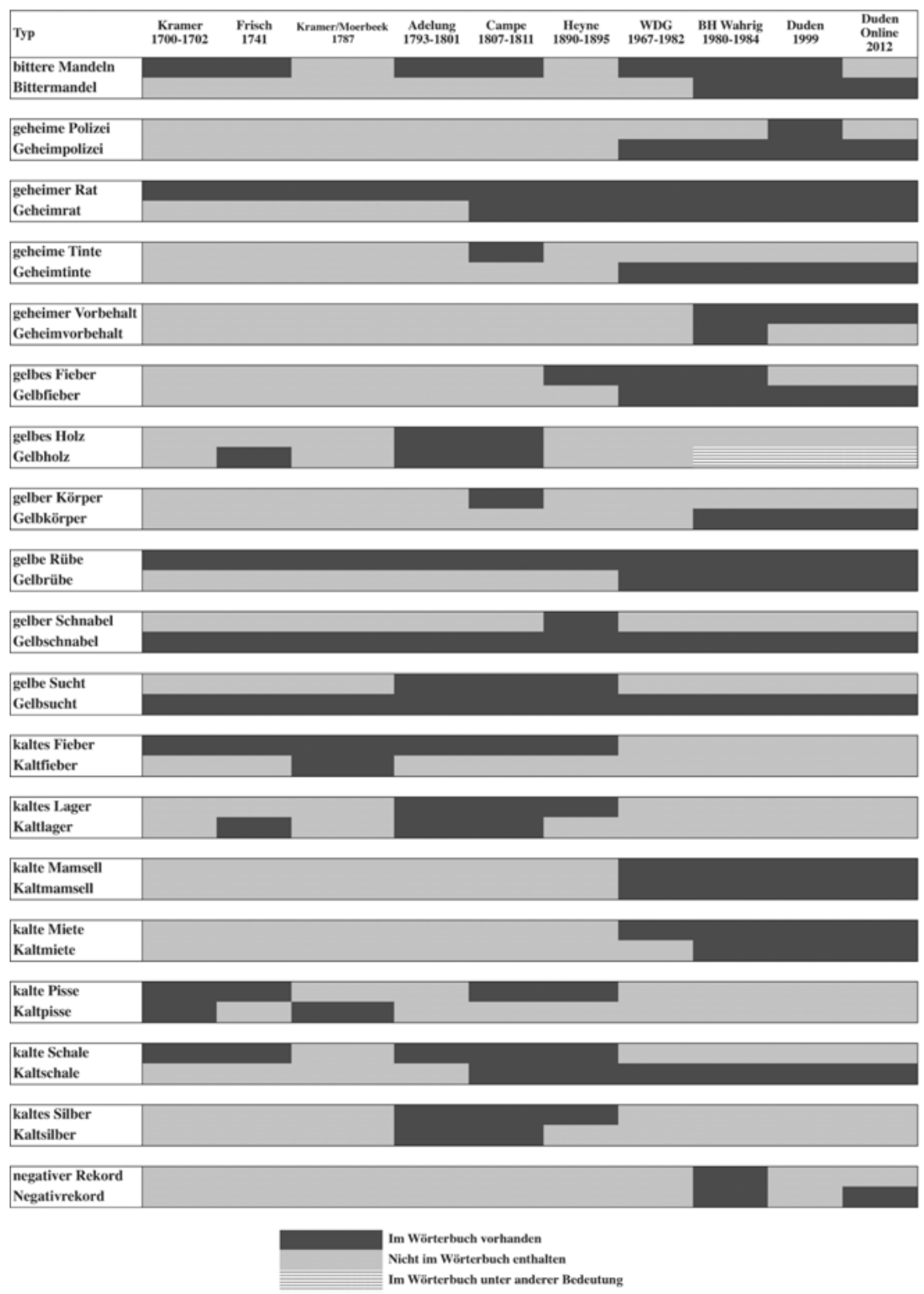




\begin{tabular}{|c|c|c|c|c|c|c|c|c|c|c|}
\hline Typ & $\begin{array}{c}\text { Kramer } \\
1700-1702\end{array}$ & $\begin{array}{c}\text { Frisch } \\
1741\end{array}$ & $\begin{array}{c}\text { Kramer/Moerbe } \\
\text { ek } \\
1787\end{array}$ & $\begin{array}{c}\text { Adelung } \\
1793-1801\end{array}$ & $\begin{array}{c}\text { Campe } \\
1807-1811\end{array}$ & $\begin{array}{c}\text { Heyne } \\
1890-1895\end{array}$ & $\begin{array}{c}\text { WDG } \\
1967-1982\end{array}$ & $\begin{array}{l}\text { BH Wahrig } \\
1980-1984\end{array}$ & $\begin{array}{c}\text { Duden } \\
1999\end{array}$ & $\begin{array}{l}\text { Duden } \\
\text { Online } \\
2012\end{array}$ \\
\hline rotes Gol & & & & & & & & & & \\
\hline Rotgold & & & & & & & & & & \\
\hline
\end{tabular}
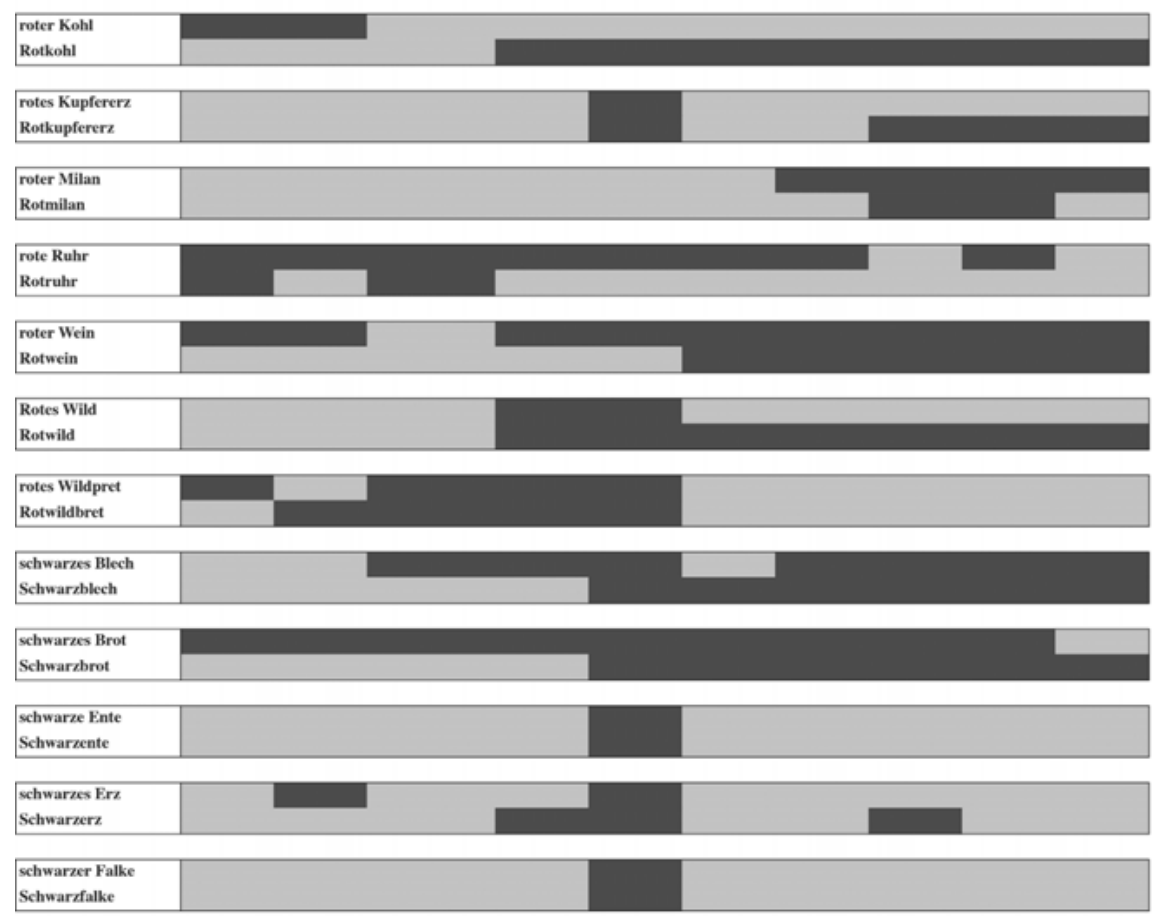

schwarzes Geld

Schwarzgeld

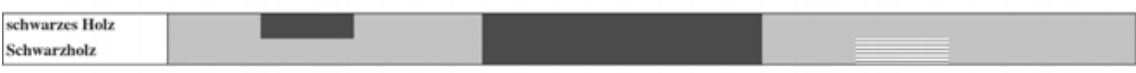

Schwarzholz

Im Wörterbuch verhanden

Nicht im Wörterbuch enthalten

Im Wōrterbuch unter anderer Bedeutung 


\begin{tabular}{|l|cccccccccc|}
\hline \multicolumn{1}{|c|}{$\begin{array}{c}\text { Kramer } \\
1700-1702\end{array}$} & $\begin{array}{c}\text { Frisch } \\
1741\end{array}$ & $\begin{array}{c}\text { Kramer/Moerbe } \\
\text { ck } \\
1787\end{array}$ & $\begin{array}{c}\text { Adelung } \\
1793-1801\end{array}$ & $\begin{array}{c}\text { Campe } \\
1807-1811\end{array}$ & $\begin{array}{c}\text { Heyne } \\
1890-1895\end{array}$ & $\begin{array}{c}\text { WDG } \\
1967-1982\end{array}$ & $\begin{array}{c}\text { BH Wahrig } \\
1980-1984\end{array}$ & $\begin{array}{c}\text { Duden } \\
1999\end{array}$ & $\begin{array}{c}\text { Duden } \\
\text { Online } \\
2012\end{array}$ \\
\hline $\begin{array}{l}\text { schwarzes Konto } \\
\text { Schwarzkonto }\end{array}$ & & & & & & & & & & \\
\hline
\end{tabular}

\begin{tabular}{|l|l|l|l|}
\hline Schwarzer Kümmel & & & \\
Schwarzkümmel
\end{tabular}
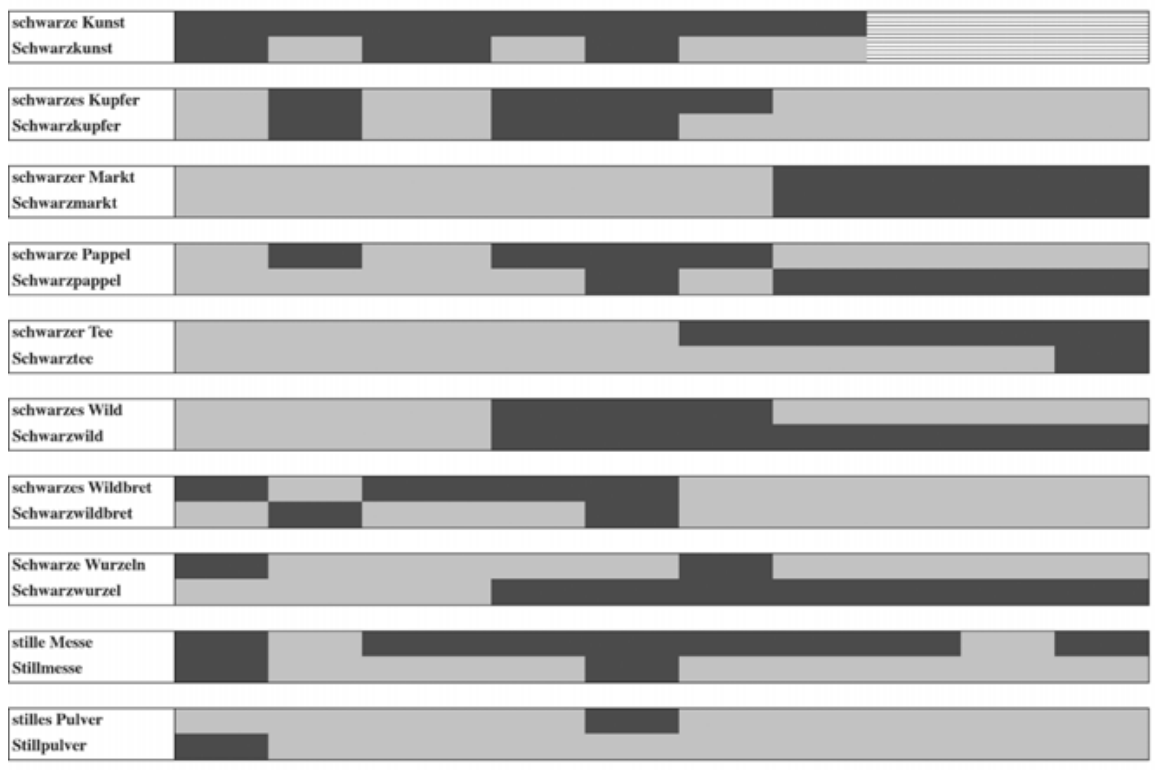

Im Wörterbuch vorhanden

Nicht im Wörterbuch enthalten

Im Wörterbuch unter anderer Bedeutung 


\section{Chronologische Übersicht zu konkurrierenden Dubletten im Primärmaterial des \\ Niederländischen}

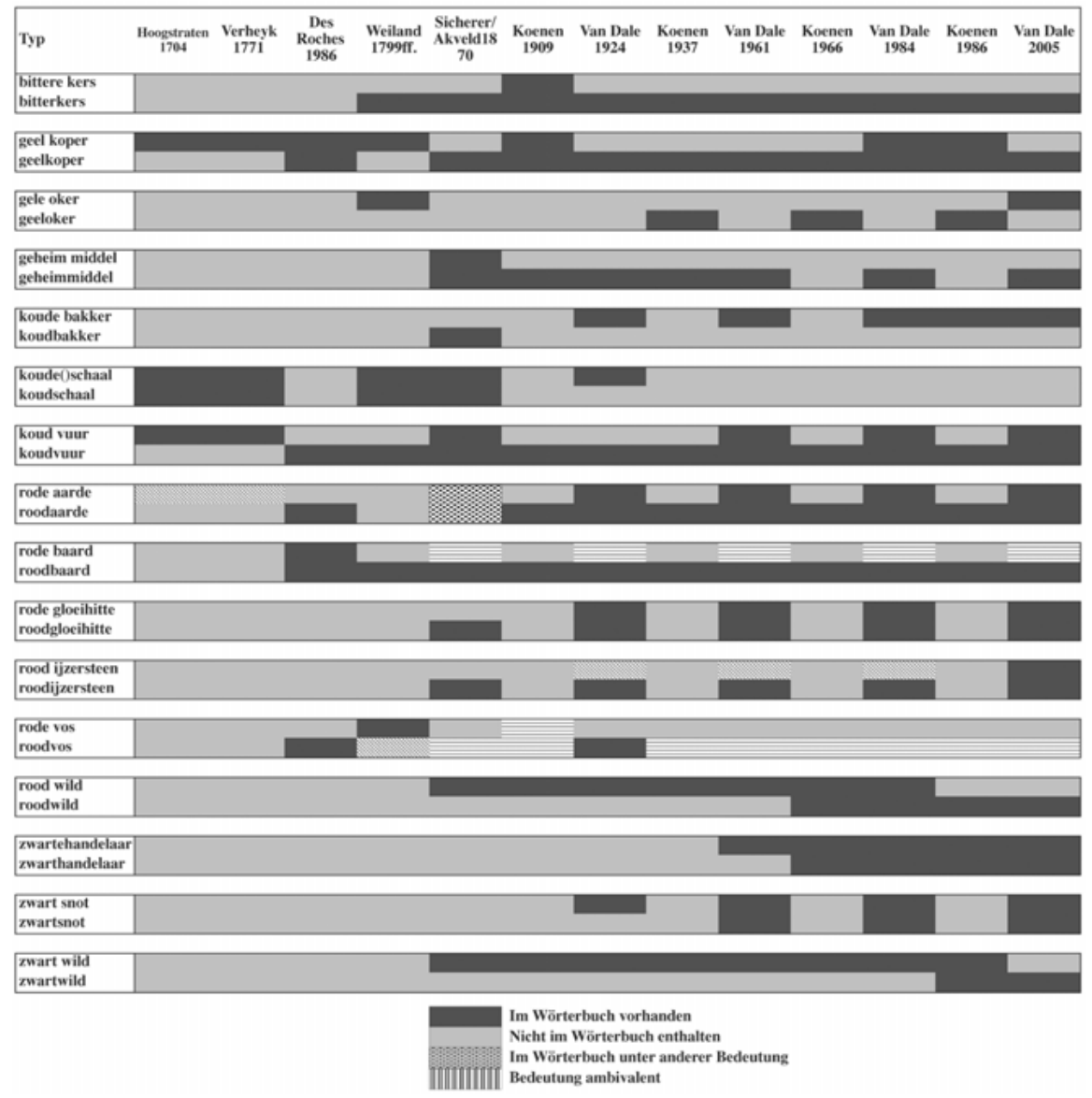

